\title{
The influence of extra-cellular matrix and stroma remodeling on the productivity of long-term human bone marrow cultures
}

\author{
Richard M. Schwartz ${ }^{1}$, Jerry Caldwel1 ${ }^{1}$, Michael F. Clarke ${ }^{2}$ Stephen G. Emerson ${ }^{2}$ and Bernhard $\emptyset$. Palsson ${ }^{1}$ \\ ${ }^{1}$ Cellular Biotechnology Laboratory, Department of Chemical Engineering; and ${ }^{2}$ Division of Hematolo- \\ gy and Oncology, Department of Internal Medicine, University of Michigan, Ann Arbor, MI 48109, USA
}

Received 9 October 1992; accepted in revised form 22 October 1992

Key words: hematopoiesis, extra-cellular matrix, stromal cells, conditioned medium, rapid perfusion

\begin{abstract}
The stromal cell layer is believed to play an important role in long-term human bone marrow cultures (LTHBMCs). At present, neither the role that the stromal cell extra-cellular matrix (ECM) plays in influencing stroma behavior is well understood nor are the effects of stroma aging. Rapid medium exchanged LTHBMCs were established on surfaces precoated with human natural fibronectin and type 1 rat tail collagen. Although initial adhesion of hematopoietic cells was improved by the presence of both ECMs, the overall progenitor and nonadherent cell productivity was not improved nor did the stroma grow to confluency faster. Thus, the ECMs used did not significantly influence the cell productivity of LTHBMCs. To examine the influence of stromal cell layer aging, conditioned medium was obtained from the first two weeks of LTHBMCs that was subsequently concentrated and used as a medium supplement in a second set of slowly exchanged LTHBMCs. The presence of the concentrated conditioned medium (conCM) enhanced the production of nonadherent cells three-fold compared with control over an eight week culture period. Control cultures that were exposed to conCM after 4 weeks in culture significantly improved their cell productivity during the latter 4 weeks of culture compared with control. The productivity of cultures exposed to conCM for 4 weeks dropped significantly when unsupplemented medium was used for the latter 4 weeks of culture. Interestingly, phytohemagglutin-stimulated leukocyteconditioned medium stimulated LTHMBCs in a similar fashion, as did conditioned medium from early LTHBMCs. Taken together, these results strongly suggest that the stromal cell layer does produce important factors for active hematopoiesis during its growth to confluence.
\end{abstract}

\section{Introduction}

Tissue Engineering is a new and growing part of biotechnology. Several studies have been carried out recently that are aimed at reconstituting functioning human tissues ex vivo. To date, perhaps the cultivation of human skin has been most successful. The development of prolific ex vivo human bone marrow systems has been long desired since such systems would enable a broad range of basic scientific and clinical studies. Applications include the study of basic dynamics of hematopoietic differentiation, improved autologous bone marrow transplantation, gene therapy of the blood cell system, and perhaps eventually the large-scale production of mature blood 
cells. Although long-term human bone marrow cultures (LTHBMCs) developed in the late 1970s and early 1980 s were initially disappointing in their longevity and cell productivity (see reviews in Greenberger 1986, Eaves et al., 1991), recent advances have markedly improved their performance.

These recent developments have used in vivo mimicry in culture in an attempt to create culture conditions that are conducive to ex vivo reconstitution of hematopoietic function. A series of studies have demonstrated that this approach is successful. The function of the supporting stromal cell layer (mostly fibroblast, with some adipocytes and endothelial cells) has been shown to be significantly influenced by the medium perfusion rate, or the medium exchange schedule. Metabolic function, growth, and perhaps most importantly growth factor secretion have all been shown to be influenced by the medium exchange rate for normal human bone marrow fibroblasts (Caldwell $e t$ al., 1991), and even for transfected NIH-3T3 murine cells (Caldwell et al., 1991a). The ability of stroma to support human hematopoiesis ex vivo has been demonstrated to be enhanced by rapid medium exchange (Schwartz et al., 1991). Under rapid medium exchange and at high cell densities, LTHBMCs can support the stable production of progenitor cells up to 20 weeks in culture and prolong granulopoiesis up to 19 weeks. The former result is notable in that it suggests strongly that these culture conditions can provide conditions suitable for stem cell maintenance and proliferation ex vivo for extended periods of time.

Judicious use of added soluble growth factors can further improve the performance of these cultures. Some hematopoietic growth factors, such as Interleukin 3 (IL-3) and GranulocyteMacrophage Colony-Stimulating Factor (GMCSF), are believed to stimulate the differentiation of early hematopoietic cells. Other growth factors, such as Erythropoietin (Epo), are believed to be terminal differentiation factors that stimulate the production of mature cells of a particular lineage. It has been shown that the addition of soluble IL-3, CM-CSF and Epo in rapidly perfused human bone marrow cultures can signifi- cantly stimulate the production of mature and progenitor cells for periods of up to 6-8 weeks (Schwartz et al., 1991a). During this period the cell culture regeneration rate (the time it takes to produce as many nonadherent cells as initially seeded) is about 2 weeks (for comparison, the estimated in vivo rate is about 2 days) and erythropoiesis is observed throughout the 20 week culture period. Both results are remarkable since all previous attempts to expand human bone marrow ex vivo have proved unsuccessful and erythropoiesis is short lived in traditional LTHBMCs (less than 2 weeks). Thus, adjustment of culture conditions to mimic the in vivo condition more closely has dramatically improved the progenitor and nonadherent cell productivity of LTHBMCs. Further, these conditions lead to the reconstitution of blood cell lineages other than the macrophagic lineage which has been observed to dominate the composition of the nonadherent cell population in LTHBMCs and bone marrow cultures from other animal species (see review in Schwartz 1991b). With the success of in vivo mimicry established, we next posed the two questions that are addressed in the present study.

First, do the extra-cellular matrices (ECMs) found in the in vivo hematopoietic environment influence the behavior of LTHMBC? The extracellular matrix plays an important role in cell adhesion (Grinnell et al., 1978; Yamada et al., 1983); cell growth (Bissell et al., 1982); and cell differentiation (Bissell et al., 1982; Coulombel et al., 1988). Endothelial cells have been shown to preferentially adhere to collagen over tissue culture plastic (Ingber et al., 1987; Ingber et al., 1989) while bone marrow progenitor cells exhibit lineage and stage-specific adhesion to extracellular matrices from marrow fibroblasts (Coulombel et al., 1988; Tsai et al., 1987; Vuillet et al., 1990). The close interaction between cells and the extracellular matrix is thus expected to influence both hematopoietic and stromal cells and thereby influence the performance of human bone marrow cultures. Therefore, if the LTHBMC microenvironment is to be modeled after the bone marrow compartment in vivo, then the in vivo ECMs should be evaluated for their effect on culture 
productivity and longevity. Collagen, fibronectin, and laminin are all native in vivo ECMs and are therefore candidates for study as ECMs in LTHBMCs. Collagen types I and III and fibronectin have been identified as major components in bone marrow fibroblast-derived ECM (Coulombel et al., 1988).

Second, does spent medium from early LTHBMCs contain unidentified growth factors that disappear in the cultures as the stromal cell layer grows to confluence? In vivo the stroma undergoes continuous remodeling, whereas in LTHBMCs the stroma grows to confluence and stromal cell proliferation ceases. Further, the cell composition of stroma changes markedly over the first few weeks of culture. The recent discovery of the kit-ligand (Zsebo et al., 1990; McNiece et al., 1991; Williams et al., 1990) and the identification of receptors on early hematopoietic cells (Mathews et al., 1991) with unknown ligands show that our understanding of the molecular control of early hematopoietic events is far from complete. Stromal cells are likely sources for many of these factors in vivo. Spent medium from early LTHBMCs could therefore contain unidentified growth factors that disappear from the culture supernatant as the stroma grows to confluence. This hypothesis can be addressed by using spent medium from early LTHBMC as conditioned medium for the entire duration of LTHBMCs.

\section{Materials and methods}

\section{Cells}

Human bone marrow cells were obtained from heparinized aspirates from the iliac crest of informed and consenting individuals. The bone marrow was separated by a Ficoll-Paque (Pharmacia, No. 17-0840-02) density gradient centrifugation and the low density cells $\left(<1.077 \mathrm{gm} / \mathrm{cm}^{3}\right)$ were collected and washed 3 times with Iscove's Modified Dulbecco's Medium (IMDM). The cells were counted between the second and third washes.
Long-term culture conditions - influence of ECM

The cells were then seeded onto: 1) 6-well tissue culture plates (Corning No. 25810) (control); 2) human natural fibronectin-coated 6-well plates (Collaborative Research Inc., Cat. No. 40402); and 3) type 1 rat tail collagen-coated 6-well plates (Collaborative Research Inc., Cat. No. 40400). All cultures were carried in duplicate and seeded at $5 \times 10^{6} \mathrm{cells} / \mathrm{ml}$ at $1.5 \mathrm{ml} /$ well.

The low density cells were incubated in IMDM supplemented with $10 \%$ fetal calf serum (Hyclone Laboratories), $10 \%$ horse serum (Hyclone Laboratories), $1 \%$ penicillin/streptomycin (Sig$\mathrm{ma}, 10,000 \mathrm{U} / \mathrm{ml}$ penicillin $\mathrm{G}$ and $10 \mathrm{mg} / \mathrm{ml}$ streptomycin, Cat. No. P3539), and $10^{-5} \mathrm{M}$ hydrocortisone (17-Hydroxy-corticosterone, Sigma, Cat. No. $\mathrm{H} 0888$ ) in a humidified $5 \% \mathrm{CO}_{2} / 95 \%$ air atmosphere. Two culture wells in the fibronectin coated plates had $0.1 \mathrm{U} / \mathrm{ml}$ of erythropoietin (Epo) added to the medium listed above, since it has been recently reported that erythroid progenitors adhere preferentially to fibronectin (Vuillet $e t$ al., 1990).

The cultures were maintained at $50 \%$ daily medium exchange. Twice per week cells were removed for counting in the medium removed during the daily medium exchange and fresh medium returned to the wells. The cells were counted using a hemocytometer and assayed for progenitor cells every fourth sampling. On days when the cells were not removed for counting, $50 \%$ of the medium was removed from each of the culture wells, the cells were centrifuged, and returned to the original wells with additional fresh medium.

\section{Preparation of conditioned medium}

Two T-150 $\mathrm{cm}^{2}$ tissue culture flasks (Corning) were each inoculated with $30 \times 10^{6}$ marrow mononuclear cells in $30 \mathrm{ml}$ of IMDM supplemented with $10 \%$ fetal calf serum (Hyclone Laboratories), $10 \%$ horse serum (Hyclone Laboratories), $1 \%$ penicillin/streptomycin (Sigma, $10,000 \mathrm{U} / \mathrm{ml}$ penicillin $\mathrm{G}$ and $10 \mathrm{mg} / \mathrm{ml}$ streptomycin, No. P3539), and $10^{-5} \mathrm{M}$ hydrocortisone 
(17-Hydroxy-corticosterone, Sigma No. H0888) [complete culture medium] in a humidified $5 \%$ $\mathrm{CO}_{2} / 95 \%$ air atmosphere. Twice weekly $100 \%$ of the medium in these cultures was replaced with $100 \%$ fresh medium. All nonadherent cells removed with the medium were discarded. Once the developing stroma reached $75-100 \%$ visual confluence (week 1), the conditioned medium (CM) removed biweekly was saved and pooled. The pooled CM was then concentrated (conCM) approximately 10-fold using an Amicon concentrator (Model 52) with a 10,000 Dalton molecular weight cut-off cellulose membrane (Millipore, No. PLGC 043 10). The medium so obtained was designated as week 1 conCM. This process was repeated the following week and medium obtained is referred to as week 2 conCM. To reduce potentially toxic levels of lactic acid and ammonia, the concentrated medium was reconstituted to its original volume with fresh medium without serum and then re-concentrated as before.

Phytohemagglutin-stimulated leukocyte conditioned medium (PHA-LCM)

Leukocytes were obtained by Ficoll-Paque density centrifugation as described above. The leukocytes were cultured at $10^{6}$ cells/ml for 7 days in the presence of $2 \mathrm{mg} / \mathrm{ml}$ phytohemagglutin. The PHA-LCM was concentrated as described above for LTHBMC medium.

\section{Culture conditions - influence of conditioned medium}

Replicate T-25 $\mathrm{cm}^{2}$ tissue culture flasks (Corning) were each inoculated with $7.5 \times 10^{6}$ marrow mononuclear cells in $5 \mathrm{ml}$ of complete culture medium (described above). Triplicate cultures were additionally supplemented with week 1 conCM, week 2 conCM, PHA-LCM, and no CM (control). The volume of concentrated conditioned medium that was added corresponded to a final concentration equivalent to $50 \%(\mathrm{v} / \mathrm{v})$ of unconcentrated CM. Twice weekly (biweekly) $100 \%$ of the medium in each culture was replaced with fresh medium. At the first weekly medium exchange, all the nonadherent cells were returned to the culture in the fresh medium. At the second weekly medium exchange, only half of the nonadherent cells were returned to the cultures (weekly demi-depletion). The cells that were not returned were used for cell counts.

\section{Methylcellulose and morphologic assays}

Once every other week the nonadherent cells removed for cell counts were plated in methylcellulose in the presence of erythropoietin and GMCSF and the Granulocyte Macrophage-Colony Forming Units (CFU-GM) were enumerated as previously described (Emerson et al., 1988). Aliquots of removed cells were occasionally cytocentrifuged, stained with Wright-Giemsa, and differential cell counts performed.

\section{Statistical analysis}

The biweekly cell production results are expressed as the mean $\pm S E M$ from replicate cultures. The probability of significant differences between groups of cultures was determined by comparing the normalized cumulative cell production values from the cultures in the collagen or fibronectin-coated plates to the matched control cultures (tissue culture plastic plates) using a paired $\mathrm{t}$-test. Statistical significance was taken at the $5 \%$ level.

\section{Results}

In the first set of experiments, the nonadherent cell production was studied in bone marrow cultures grown in collagen-coated, fibronectin-coated, or similar uncoated $35 \mathrm{~mm}$ tissue culture dishes. The biweekly cell production of all cultures showed the usual rapid decay in nonadherent cell production during the first 2 weeks in culture followed by approximately 4 weeks of stable cell production in all cultures (see Schwartz 1991). The cumulative number of cells produced in the collagen and fibronectin-coated culture dishes was not 


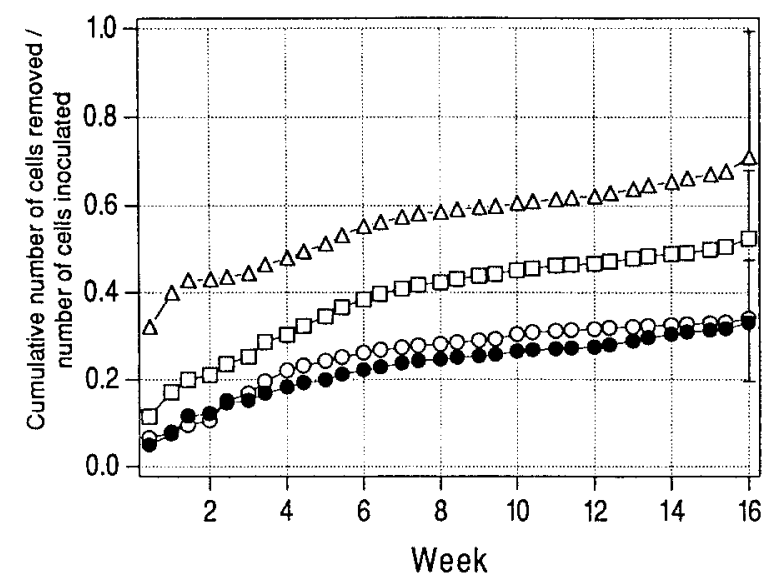

Fig. 1. The normalized cumulative number of nonadherent cells produced in LTBMCs on different ECMs following the first sampling; tissue culture plastic $(\Delta)$ (control), collagen type I ( $\square$ ), fibronectin (O), or fibronectin with Epo in the medium $(O)$. Confidence intervals are the cumulative \pm SEM at 16 weeks of culture.

significantly different than those produced in the corresponding tissue culture plastic cultures at the 10\% level of significance (Fig. 1).

Light microscopic observations were used to qualitatively evaluate the growth of the stromal layer during the initial two weeks in culture when stromal cells rapidly proliferate. Our observations revealed that the time required for the stromal layer to reach confluency was essentially independent of the ECM used.

The extracellular matrix does appear to have an effect on the initial adhesion of the bone marrow cells to the ECM. The number of cells removed during the initial sampling of the cultures at day 3 was $2.7,6.2$ and 4.7-fold higher in the plastic dishes than in the dishes coated with collagen, fibronectin, or fibronectin with Epo in the medium. However, a statistical analysis of the number of cells removed in the first sampling of cells from the cultures shows no statistical difference between the tissue culture plastic and the other ECMs. This lack of statistical significance may be due to a low number of samples (2).

Hematopoietic progenitor cell (CFU-GM, BFU-E, and CFU-GEMM) assays were performed from samples removed from each LTHBMC (Table 1). Similar to previous results obtained under this protocol (Schwartz 1991), progenitor cell production was maintained at high levels in this experiment as seen by CFU-GM production through 16 weeks in culture. The cultures on plastic appeared to maintain a more constant production level of CFU-GM than did the cultures with precoated collagen or fibronectin surfaces. The cumulative number of CFU-GM removed through 16 weeks, is higher in the cultures grown on collagen due to higher CFU-GM

Table 1. The average number of progenitor cells removed from LTBMCs cultures on different extracellular matrices

\begin{tabular}{|c|c|c|c|c|c|c|c|c|c|c|c|c|}
\hline \multirow[b]{2}{*}{ Week } & \multicolumn{3}{|l|}{ Plastic } & \multicolumn{3}{|l|}{ Collagen } & \multicolumn{3}{|c|}{ Fibronectin } & \multicolumn{3}{|c|}{ Fibronectin + Epo } \\
\hline & BFU-E & $\begin{array}{l}\text { CFU- } \\
\text { GM }\end{array}$ & $\begin{array}{l}\text { CFU- } \\
\text { GEMM }\end{array}$ & BFU-E & $\begin{array}{l}\text { CFU- } \\
\text { GM }\end{array}$ & $\begin{array}{l}\text { CFU- } \\
\text { GEMM }\end{array}$ & BFU-E & $\begin{array}{l}\text { CFU- } \\
\text { GM }\end{array}$ & $\begin{array}{l}\text { CFU- } \\
\text { GEMM }\end{array}$ & BFU-E & $\begin{array}{l}\text { CFU- } \\
\text { GM }\end{array}$ & $\begin{array}{l}\text { CFU- } \\
\text { GEMM }\end{array}$ \\
\hline 2 & 7 & 45 & 1.5 & 5 & 73 & 2 & 7 & 62 & 1 & 9 & 61 & 2 \\
\hline 4 & 10 & 57 & 1.5 & 2 & 66 & 0 & 14 & 43 & 0.5 & 5 & 28 & 0 \\
\hline 6 & 1 & 22 & 0.5 & 3 & 39 & 2 & 6 & 34 & 2.5 & 2 & 16 & 0.5 \\
\hline 8 & 0 & 35 & 3 & 1 & 89 & 3.5 & 0 & 29 & 0.5 & 0 & 33 & 1.5 \\
\hline 10 & 1 & 64 & 0 & 2 & 71 & 0.5 & 1 & 49 & 3 & 0 & 12 & 1.5 \\
\hline 12.5 & 0 & 25 & 0 & 0 & 19 & 0 & 1 & 23 & 0 & 0 & 6 & 0 \\
\hline 14 & 2 & 30 & 0 & 0 & 12 & 0 & 0 & 8 & 0 & 0 & 0 & 0 \\
\hline 16 & 0 & 29 & 0 & 0 & 4 & 0 & 0 & 1.5 & 0 & 0 & 25 & 0 \\
\hline Total & 21 & 307 & 6.5 & 13 & 373 & 8 & 29 & 249.5 & 7.5 & 16 & 181 & 4.5 \\
\hline
\end{tabular}

Progenitor cells were assayed once every other week (every fourth culture sampling). 
production during the first 8 weeks in culture. Cumulative BFU-E production is highest in fibronectin cultures without Epo. However, statistical analysis of the cumulative CFU-GM, BFU-E, and CFU-GEMM production through 16 weeks in culture shows that neither the collagen nor the fibronectin ECM was statistically different from control at the $5 \%$ level of significance using a t-test.

In the second set of experiments, slowly-exchanged LTHBMCs were exposed to conditioned media for an eight week time period. Four different protocols were run: (1) use of 1 week conCM throughout the 8-week period, (2) use of PHALCM throughout the 8-week period, (3) use of week 2 conCM for 4 weeks followed by 4 weeks of regular medium, (4) use of regular medium for 4 weeks followed by the use of week 2 conCM for 4 weeks, and (5) use of regular medium throughout (control). The cumulative number of cells collected throughout the eight-week period differed significantly between the five protocols (Fig. 2). The control culture showed a steady decline in the nonadherent cell numbers harvested until week 8, and there is no significant addition to the cumulative number of cells collected during the last weeks of the culture. This pattern is

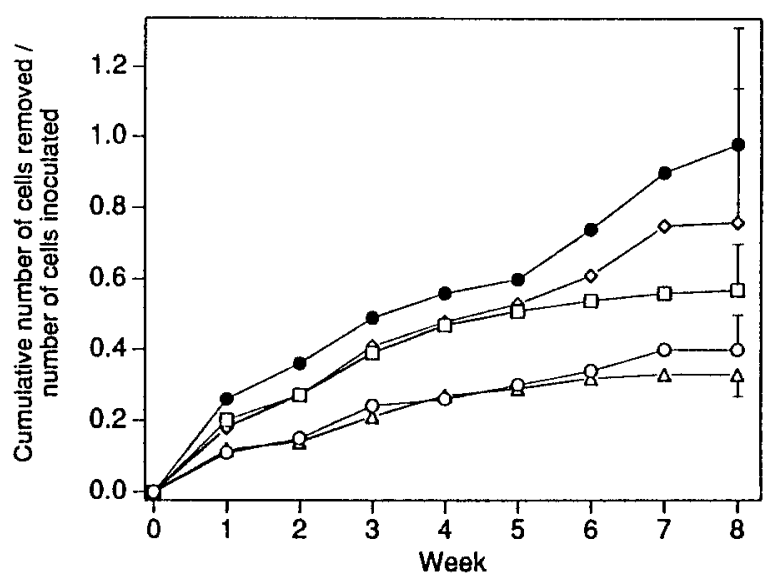

Fig. 2. The normalized cumulative number of nonadherent cells produced in LTBMCs using different conditioned medium: control $(\Delta)$, conCM throughout $(0)$, conCM through week $4(\theta)$, conCM following week $4(\mathrm{O})$, and PHA-LCM $(\Theta)$. Confidence intervals are the cumulative \pm SEM at 8 weeks of culture. typical of traditional slowly-exchanged LTHBMC (e.g., Greenberger 1986). The cultures that were cultivated in either the week 1 conCM or the PHA-LCM throughout outperformed the control culture by a factor of three in terms of nonadherent cell produced and were still actively producing cells at the end of the eight week period. Using a t-test, statistical analysis of the cumulative cell production data for all protocols, when compared the control cultures, were significant at the $99 \%$ confidence level.

Protocols (3) and (4) exhibited intermediate cell production. Culture number (3) produced similar cell numbers as the culture cultivated in the week 2 conCM and then following the switch at week 4 to regular medium the culture decreased its production of nonadherent cells to the same levels as observed for the control culture. Likewise, culture (4) produced similar cell numbers as the control culture until week 4 and then after being cultivated in the week 2 conCM became more prolific with respect to nonadherent cell production and resembled that of the culture that was cultivated in week 1 conCM throughout.

\section{Discussion and conclusions}

Although the stromal layer is believed to be required to maintain long term hematopoiesis, neither the role the stromal cell ECM plays nor the effects of its aging is well understood.

The data presented here suggests that the rate of attachment of stromal cells to the ECM and the time required to reach confluency was independent of the ECM used. This result suggests, then, that the ECM does not significantly influence bone marrow fibroblasts, the primary stromal cell type. Adhesion of hematopoietic cells to the ECM may be important early in culture development before the stromal cells have time to grow and provide an adherent surface for the hematopoietic cells. Adhesion of primitive hematopoietic cells is crucial to culture productivity and longevity because removal of nonadherent primitive hematopoietic progenitor cells will cause a significant loss of proliferative potential (Varma et al., 
1991). After the stroma is developed (1.5-2 weeks), the effect of the ECM becomes less important as the stroma deposit their own ECM and the hematopoietic cells have no direct contact with the original ECM. Interestingly, these data suggest that although initially hematopoietic cells adhere better to collagen- and fibronectin-coated surfaces than to plastic, the performance of the long-term culture is not significantly influenced.

The data from the first set of experiments show that the ECMs used in this study do not improve the productivity of rapidly-exchanged LTHBMCs. Further, these data closely duplicate results reported recently of slowly-exchanged traditional LTHBMCs grown on different ECMs (Mergenthaler et al., 1990) which demonstrated that the ECM had little effect on stromal growth early in culture and hematopoietic progenitor and mature cell production through 8 weeks in culture. Therefore, the requirement of stromal cells for the maintenance of LTHBMCs must be greater than providing ECM.

Hematopoietic growth factors may be more important to the establishment of prolific LTHBMCs than provision of an ECM. Such growth factors are believed to be provided, in part, by the stromal cells. The stromal cells grow to confluence typically in 2 to 3 weeks in LTHBMCs with little subsequent proliferation. The results from our second set of experiments strongly'suggest that the ability of the stroma to support hematopoiesis does change with time in LTHBMCs. The use of week 1 conCM (culture (1)) led to a cell production rate that was superior to the control culture (5). Cultures supplemented with week 2 conCM (3), when taken off the conditioned medium, abruptly exhibited decreased nonadherent cell productivities to resemble that of the control culture (5). Interestingly, unsupplemented cultures (4) when exposed to the week 2 conCM after four weeks, experienced significant stimulation. This result is significant and suggests that the loss of productivity in LTHBMCs may not be irreversible but due to the loss of important stimulatory factors. An alternative explanation, however, for the increase in cell production is that it is due to an increase in serum factors, which resulted from concentrating the serum-containing culture medium. This possibility is unlikely in that studies undertaken to determine an optimum serum concentration and composition for human LTBMCs, have shown that 20-25\% serum (50\% each bovine and horse serum) to be most effective for culture longevity and productivity (Gartner and Kaplan, 1980; Greenberg et al., 1981). The conclusion that one arrives at from this data is that culture conditions for LTHBMCs need to be adjusted so that the function of the stromal cell layer is maintained over the duration of the culture, possibly through continuous remodeling.

\section{References}

Bisseli MJ, Hall HG and Parry G (1982) How does the extracellular matrix direct gene expression? J. Theor. Biol. 99: 31-68.

Caldwell J, Locey B, Clarke MF, Emerson SG and Palsson BØ (1991) The influence of medium exchange schedules on metabolic, growth, and GM-CSF secretion rates of genetically engineered NIH-3T3 cells. Biotechnology Progress 7: 1-8.

Caldwell J, Palsson B $\emptyset$ and Emerson SG (1991a) Culture perfusion schedules influence the metabolic activity and granulocyte-macrophage colony-stimulating factor production rates of human bone marrow stromal cells. J. Cell. Physiol. 147: 344-353.

Coulombel L, Vuillet MH, Leroy C and Tchernia G (1988) Lineage- and stage-specific adhesion of human hematopoietic progenitor cells to extracellular matrices from marrow fibroblasts. Blood 71(2): 329-334.

Eaves CJ, Cashman JD and Eaves AC (1991) Methodology of long-term culture of human hematopoietic cells. J. Tiss. Cult. Meth. 13: 55-62.

Emerson SG, Yang YC, Clarke SC and Long MW (1988) Human recombinant GM-CSF and IL-3 have overlapping but distinct hematopoietic activities. J. Clin. Invest. 82: 12821287.

Gartner S and Kaplan HS (1980) Long-term culture of human bone marrow cells. Proc. Natl. Acad. Sci. USA 77: 47564759.

Greenberg HM, Newburger PE, Parker LM and Greenberger JS (1981) Generation of physiologically normal peripheral blood granulocytes in continuous bone marrow cultures. Blood 58(4): 724-731.

Greenberger JS (1984) Long-term hematopoietic cultures. In: Methods of Hematology, Vol. 11: (pp. 203-242) Churchill Livingstone.

Grinnell F (1978) Cellular adhesions and extracellular substrata. Int. Review. Cytol. 53: 65-144. 
Ingber DE, Madri JA and Folkman J (1987) Endothelial growth factors and extracellular matrix regulate DNA synthesis through modulation of cell and nuclear expansion. In Vitro Cell. Devel. Biol. 23(5): 387-394.

Ingber DE and Folkman J (1989) How does extracellular matrix control capillary morphogenesis? Cell 58: 803-805.

Mathews W, Jordan T, Waigand GW, Pardoll D and Lemischka IR (1991) A receptor tyrosine kinase specific to hematopoietic stem and progenitor-enriched cell populations. Cell 65: 1143-2252.

McNiece IK, Langley KE and Zsebo KM (1991) Recombinant human stem cell factor synergises with GM-CSF, G-CSF, IL-3 and Epo to stimulate human progenitor cells of the myeloid and erythroid lineages. Exp. Hematol. 19(3): 226231.

Mergenthaler H-G and Dormer P (1990) Hemopoiesis in human micro long-term bone marrow culture with preformed extracellular matrix. Haematologica 75: 12-16.

Schwartz RM, Palsson B $\emptyset$ and Emerson SG (1991) Medium perfusion rate significantly alters the cell productivity and longevity of human bone marrow cultures. Proc. Natl. Acad. Sci. USA 88: 6760-6764.

Schwartz RM, Palsson B $\varnothing$ and Emerson SG (1991a) In vitro myelopoiesis stimulated by rapid medium exchange and supplementation with hematopoietic growth factors. Blood 78(12): 3155-3161.
Schwartz RM (1991b) Optimization of Human Long-term Bone Marrow Cultures. PhD Thesis, The University of Michigan, Ann Arbor.

Tsai S, Patel P, Beaumont E, Lodish HF, Nathan DG, and Sieff CA (1987) Differential binding of erythroid and myeloid progenitors to fibroblasts and fibronectin. Blood 69(6): 15871594.

Varma A, El-Awar FY, Palsson B $\varnothing$, Emerson SG, and Clarke MF (1990) Can Dexter cultures support stem cell proliferation? J. Exp. Hematol. 20: 87-91.

Vuillet-Gaugler MH, Breton-Gorius J, Vainchenker W, Guichard J, Tchernia G and Coulombel L (1990) Loss of attachment to fibronectin with terminal human erythroid differentiation. Blood 75(4): 865-873.

Williams DE, Eisenman J, Baird A, Ranch C, Van Ness K, March CJ, Park LS, Martin U, Mochizuki DY and Boswell HS (1990) Identification of a ligand for the c-kit protooncogene. Cell 63(1): 167-174.

Yamada KM (1983) Cell surface interactions with extracellular materials. Ann. Rev. Biochem. 52: 761-799.

Zsebo KM, Wypych J, McNiece IK, Lu HS, Smith KA, Karkare SB, Sachdev RK, Yuschenkoff VN, Birkett NC and Williams LR (1990) Identification, purification, and biological characterization of hematopoietic stem cell factor from buffalo rat liver-conditioned medium. Cell 63(1): 195-201.

Address for offprints: Bernhard $\varnothing$. Palsson, Cellular Biotechnology Laboratory, Department of Chemical Engineering, University of Michigan, Herbert H. Dow Building, Ann Arbor, MI 48109, USA 